\title{
Factores psicosociales en la construcción del conocimiento prototípico de las emociones
}

\author{
Darío Paez y Anabel Vergara \\ Universidad del País Vasco
}

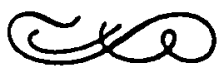

\begin{abstract}
Resumen
Este articulo presenta una investigación sobre prototipos emocionales y su asociación con las relaciones sociales y el sexo de los sujetos. Concepciones de sentido común de rabia, tristeza y alegría se infirieron a partir de narraciones de episodios emocionales. El reconocimiento de la tipicalidad de los atributos y su recuerdo libre categorizado, se encontraron asociados al sexo (las mujeres mostraban más expresividad emocional y sensibilidad subjetiva, así como mayor sensibilidad a los antecedentes y efectos sociales de las emociones) y a la densidad de relaciones sociales (los sujetos con un mayor número de roles mostraban mayor recuerdo y tipicalidad de los antecedentes sociales, de formas de manejo emocional y tenían una experiencia emocional más rica). El soporte social objetivo y subjetivo, también influenciaban el conocimiento social de las emociones. Escenario o guiones emocionales se extranjeron de las narraciones mediante un análisis factorial de correspondencias $y$ las puntuaciones totales de los atributos asociados a cada factor (representando los escenarios) tuvieron asociaciones significativas con el sexo y con el nivel de relaciones sociales - particularmente con el soporte social subjetivo-. Concluimos que el número de roles, el soporte social objetivo y subjetivo, así como el sexo, influencial el contenido y la estructura de los prototipos y escenarios emocionales.
\end{abstract}

Palabras clave: Prototipos emocionales.

\section{Psychosocial variables in the prototypical knowledge of emotions}

\section{Abstract}

This article presents a study on Emotional Prototypes related to the level of social relationships and sex of subjects. The sample was composed of 110 adults -Basque Country resident nonstudents. Lay conceptions of anger, sadness and joy were inferred from narratives of emotional episodes. The prototypicality and free recall ratings of features were related to sex (women have more emotional expressiveness and greater sensitivity to internal emotional events and to external social antecedents and effects of emotion) and to density of social relationships (subjects with a bigh number of roles showed greater recall and prototypicality of social antecedents, of ways of bandling emotions, and bad a richer experience of emotion). Subjective and objective social support also influences the social knowledge of emotion. Emotional scripts were exacted with a correspondence factor analysis and the factor total scores have significative associations with sex and social relationship variables-particularly with subjective social support. We concluded that the number of roles, objetive and 'subjective social support, and sex affect the content and structure of people's emotion prototypes and scripts.

Keywords: Emotional prototypes.

Dirección del autor: Dpto. de Psicología Social y Metodología. Universidad del País Vasco. Apdo. 1249. Donostia. 


\section{INTRODUCCION}

Toda una línea de investigaciones sobre las emociones se desarrolla, conceptualizando a éstas como categorías naturales de conocimiento social, en otros términos, como esquemas o prototipos de conocimiento social sobre las emociones. Dentro de esta línea, el estudio de las cogniciones como proposiciones interrelacionadas expresadas lingüísticamente, es básico. Diferentes autores han planteado el estudio de los escenarios prototípicos o de los esquemas cognitivos (véase las teorías de Lang e Isen en Paez, Echebarría y Villareal, 1989, por ejemplo) o de los conceptos naturales de conocimiento social sobre las emociones, basados en el lenguaje (Averill, 1982; Shaver et al., 1987). Averill fue uno de los primeros en sugerir que los conceptos del lenguaje cotidiano son categorías naturales de conocimiento, que forman un sistema jerárquico. Por ejemplo, el enojo sería una categoría emocional básica del lenguaje y del conocimiento social (Averill, 1982).

Categorías naturales prototípicas como formas de conocimiento social de las emociones

Desarrollando esta orientación, algunos autores han buscado, a partir de las narraciones de episodios emocionales y/o de las descripciones por sujetos legos de los atributos de las emociones, inferir los conceptos de sentido común o las categorías naturales sobre las emociones. La orientación de los conceptos naturales como prototipos, elaborado por Rosch et al., se ha extendido al campo de la cognición social de las emociones. Un prototipo se define como un conjunto abstracto de rasgos o atributos generalmente asociados a los miembros de una categoría. Cada rasgo se pondera por su grado de asociación a la categoría. Una categoría se concibe como el conjunto de objetos que son considerados equivalentes (Semin, 1988).

Estas categorías emocionales se formarían a partir de experiencias repetitivas. Su proceso de aprendizaje sería el de la similaridad entre sucesos episódicos, y se basaría en las estructuras reales de asociación entre atributos. Estas categorías emocionales se organizarían alrededor de prototipos (ya sea ejemplares o episodios prototípicos reales o abstracciones ideales de éstos). Estos conceptos estarían definidos de manera difusa por atributos, ni suficientes ni necesarios. Los atributos más importantes para definir una categoría emocional son las propiedades máximas o ideales de una categoría emocional. Un prototipo sería el ejemplar o el tipo ideal que reuniría el máximo de atributos asociados a una categoría dada y el mínimo de atributos asociados a otras categorías.

La teoría de las categorías conceptuales prototípicas, postula la existencia de una jerarquía vertical o taxonómica de los conceptos emocionales. Presupone que habrían tres niveles conceptuales en el conocimiento social de las emociones: un nivel intermedio o básico, un nivel supraordenado más general y un nivel subordinado más concreto. El nivel básico se caracterizaría por aprenderse primero en la adquisición del léxico emocional; sería más accesible ante estímulos relevantes; es más probable que esté asociado a etiquetas verbales breves y serían las categorías más abstractas que se pueden representar por 
una imagen (Fehr, 1988). Esto fue confirmado por la investigación de Shaver et al. (1987) que encontró como categorías supraordenadas las de afectividad positiva y negativa, y como categorías básicas el miedo, la alegría, la tristeza, el amor y la sorpresa - además del enojo o rabia-. Los nombres de las seis emociones básicas se eligieron a partir de las palabras de mayor prototipicalidad. Las seis emociones mencionadas coinciden con las listas de emociones básicas de autores neo-darwinistas como Ekman (menos asco, que está en la de Ekman y amor que no está) y otros autores. Además, estas emociones se adquieren primero en aprendizaje verbal: los niños de cuatro años mencionan espontáneamente feliz, triste, loco («mad» o enojado) y asustado, aunque pueden hablar de gustar (like) o amor (Shaver et al., 1987). Igualmente, son las emociones que tienen nombre en la mayoría de los léxicos emocionales, según indica la investigación antropológica (Lutz, 1988). Por otro lado, la teoría de los conceptos prototípicos postula una relación entre la tipicalidad de los atributos o ejemplos de una categoría emocional, el recuerdo y el juicio. Los atributos o ejemplos más típicos, se recordarían más, se evaluarían como pertenecientes a la categoría más rápidamente, se darían más como buenos ejemplos, etc. Fehr (1988) encontró un relación significativa entre la tipicalidad de los atributos del amor y su tasa de recuerdo libre en otra muestra $(r=.38)$. En una serie de investigaciones, replicando los prototipos de Shaver et al. (véase Paez y Vergara, 1990) hemos podido constar que la fiabilidad de las puntuaciones de tipicalidad son todas significativas, estimada mediante la correlación entre las medidas de tipicalidad de los atributos en dos muestras -y salvo la de miedo que era de .66, las otras tipicalidades eran altas (tristeza, .86; alegría, .96; y cólera, .92). A nivel de la relación entre recuerdo libre y puntuaciones de tipicalidad, vemos que la relación entre recuerdo libre autocategorizado, tiene relaciones significativas con las de tipicalidad -cólera y tristeza tienen las relaciones más fuertes $(.89$ y .79), siendo las de alegría y miedo medianas $(.70$ y .78) - Esto confirma la relación entre el recuerdo y la centralidad del atributo en relación al prototipo. La validez transcultural de estos resultados, se ven reafirmados por las relaciones significativas entre las medias de recuerdo libre de la muestra de Shaver et al., y nuestros resultados de tipicalidad -correlaciones de .37 para cólera, de .33 para alegría, de .50 para tristeza y de .58 para miedo-. Por último, se plantea la existencia de ciertos atributos compartidos por los sujetos de una cultura en lo referente a la definición de un concepto. Según Averill, los atributos prototípicos de una emoción serían las respuestas modales de los sujetos. Salmaso y Pombeni (1986), Cantor et al. (in Ginsburg, 1988) así como Shaver et al., utilizaron el criterio del $20 \%$ del recuerdo libre y Fehr el criterio del $2 \%$. En nuestra réplica de la investigación de Shaver et al: (Paez y Vergara, 1990) confirmamos que se puede extraer un cierto núcleo común estable, que caracteriza a los atributos narrados de episodios emocionales - un $50 \%$ de los atributos de las cuatro emociones básicas, descritas por Shaver et al. en una muestra norteamericana de estudiantes, se replicaron como atributos centrales en muestras de estudiantes o familiares de estudiantes del País Vasco-.

Pese a esto, se pueden criticar ciertas bases de la aplicación de la teoría de las categorías prototípicas, al lenguaje emocional: se afirma que el hecho de que la gente sea capaz y encuentre psicológicamente significativo el calificar a unas palabras emocionales o a unos atributos como más prototípicos que 
otros, confirma el carácter difuso y la existencia de una jerarquía vertical y horizontal. Sin embargo, aún en relación a conceptos con criterios de definición necesarios y suficientes, claramente delimitados, como los numeros pares e impares, se ha encontrado que hay mejores ejemplares - los números 2, 4 y 8 son evaluados como mejores ejemplares de pares que los números 34, 106 ó 806, por ejemplo (Clore y Ortony, 1988). Igualmente, la existencia de condiciones necesarias y suficientes no implica la ausencia de grados de certeza o de indeterminación en relación a casos fronterizos (op. cit. ant.). Hay autores que diferencian la existencia de un núcleo definitorio «clásico», es decir, que daría los atributos necesarios y suficientes lógicamente para una clase, y la existencia de procedimientos difusos y probabilísticos, que ayudaría a identificar a un episodio emocional como perteneciente a una categoría emocional dada mediante heurísticos, y que provocarían efectos de tipicidad (Pozo, 1988). Según el ejemplo clásico, una abuela lo sería única y exclusivamente por tener nietos (núcleo definitorio), aunque existe un proceso de identificación probabilístico de clasificación, basado en atributos perceptivos y funcionales, dados en diversa medida por los ejemplares (tener arrugas, ser mayor de edad, cabello blanco, etc.) (Bellelli, 1987). En el caso de las emociones, habría un núcleo definitorio, que según los construccionistas sociales serían los atributos de activación fisiológica, vivencia de pasión o descontrol, situación causal, y tendencia de acción unida a normas sociales (de bloqueo o injusticia y búsqueda de reparación o superación de la norma rota o bloqueada en el caso del enojo); mientras que la identificación se asociaría a atributos variables (expresión abierta o no del enojo, presencia de agresión verbal, física, elementos específicos de activación, etc.).

Otra crítica que se plantea, es que no se puede deducir automáticamente de un análisis de narracioneș y del lenguaje emocional, de su jerarquía, metáforas y reglas proposicionales, conclusiones psicológicas válidas -ésta es una versión particular de la crítica general al reduccionismo lingüístico(Lakoff \& Kovecses, 1987). El significado de una categoría natural de conocimiento social sobre las emociones, no es reducible a una lista de atributos $-\mathrm{y}$ de hecho lo que Shaver et al. generaron, fueron más bien escenarios del tipo antecedentes-desarrollo-desenlace. Esto se vincula además, a la limitación de la concepción de formación de estas categorías naturales. Según Rosch, se formarían mediante la observación de relaciones de asociación reales entre atributos y entre la presencia de atributos y la pertenencia a una categoría emocional dada. Esta concepción del aprendizaje de las categorías naturales de las emociones mediante un mecanismo asociativo de detección de contingencias y de abstracción de episodios, no resuelve el problema de la selectividad de atributos, ni el de la taxonomía de categorías. Estos procesos se pueden explicar, sólo por la existencia de teorías implícitas distribuidas culturalmente (Pozo, 1989).

Conocimiento social sobre las emociones, construccionismo social y lenguaje

El hincapié puesto por autores como Averill, en el análisis del lenguaje y del conocimiento social sobre las emociones, se basa en presupuestos del 
costruccionismo social. Para esta corriente de psicología social, las emociones son constructos de sentido común "multireferenciales». Una emoción es un significado aprendido que le permite al sujeto, organizar una experiencia privada. Este constructo está asociado semánticamente a una serie de creencias interpretativas. Metafóricamente, la gente internaliza y objetiviza este discurso, pero, lo esencial es no dejarse llevar por esta reificación de las emociones y estudiar las prácticas sociales y condiciones históricas que ayudan a crear un significado emocional a un estado (Hallam, 1985).

Además de asociaciones lingüísticas cuasi-automaticas, las personas aprenden y generan creencias populares, folklóricas, de sentido común. Estas teorías informales o representaciones sociales, también van a influir la conducta emocional. Varios teóricos cognitivistas que habían planteado concepciones prototípicas de las categorías de conocimiento natural, insisten actualmente en la relevancia de las teorías folklóricas implícitas, para comprender el funcionamiento de éstas. Por ejemplo Medin, co-autor de la teoría prototípica de las categorías naturales, ha planteado que las categorías o conceptos naturales no tendrían un núcleo de atributos definicionales, sino que éstos serían cambiantes, dependientes del contexto y basados en las experiencias. Empíricamente, esto se ve reafirmado por el hecho de que la perspectiva de generación del conocimiento influencia la representación de la experiencia emocional. Shaver et al. (1987), encontraron que los sujetos mencionaban más frecuentemente los sesgos y distorsiones cognitivas, cuando describían la experiencia emocional de una persona típica, que cuando describían la suya propia. Segundo, cuando describían sus propias emociones, mencionaban más a menudo sus predisposiciones personales, sus interpretaciones, sus esfuerzos de autocontrol. En cambio, cuando describían la experiencia emocional de una persona típica, mencionaban más sus reacciones expresivas y conductás manifiestas, replicando las diferencias clásicas entre actor-observador.

Se ha encontrado igualmente, inestabilidad en las tareas de estimación de tipicalidad sobre objetos naturales: la correlación entre cómo ordenan todos los sujetos de una muestra los ejemplares de una categoría por tipicalidad - según los procedimientos de Rosch- es de .5 , indicando que sólo hay un $25 \%$ de la varianza compartida. La misma correlación, intrasujetos, es de .78 , indicando que sólo hay un $61 \%$ de la varianza compartida (Barsalou y Medin, 1986). Esto ha llevado a algunos autores, entre ellos Medin, a descalificar esta aproximación (Oden, 1987). Se ha planteado que las categorías o conceptos naturales, no tendrían un núcleo de atributos deficionales, sino que éstos serín cambiantes, dependientes del contexto y basados en las experiencias. Ahora bien, estos conceptos, categorías o esquemas, no se basarían sólo en las experiencias, sino que también estarían basados en teorías intuitivas, implícitas, compartidas culturalmente, que explicarían la estructura y funciones de los atributos de una categoría - en este caso emocional-. Estas teorías seleccionarían, interpretarían y organizarían las propiedades de las categorías en su proceso de formación (Barsalou y Medin, 1986). Concepciones similares son planteadas por antropólogos que han estudiado el léxico emocional. Lutz (1988), plantea que a partir del estudio de la sintaxis, metáforas y redes proposicionales del léxico, se pueden inferir modelos conceptuales. Estos conceptos están implicados en una red integral de imágenes y proposiciones. En cada comunidad existen ciertos escenarios emocionales que se pueden calificar como prototípicos. Por ejemplo, entre los ifaluk el 
enojo justificado implica una clara transgresión por parte de un sujeto, transgresión identificada de manera calma por otra persona, que muestra una expresión facial adusta, ante la cual la primera persona responde con miedo/ansiedad. Ahora bien, el léxico emocional juega no sólo un rol referencial, de categorización o identificación de situaciones emocionales, sino que también juega un rol retórico - se usa para impulsar a los otros a la acción o para defender la autoestima- (Lutz, 1988). Lo relevante es que, tanto psicólogos cognitivos como antropólogos, han planteado tres elementos centrales de las aproximaciones construccionistas sociales de las emociones: la centralidad del léxico emocional para el estudio de las emociones; la dependencia de los conceptos prototípicos y escenarios implícitos en el lenguaje de las representaciones culturales y; el carácter pragmático, orientado al uso social del léxico emocional. Ahora bien, esto no niega que haya elementos interesantes en la aproximación al conocimiento social de las emociones como categorías naturales prototípicas.

Respecto a este conjunto de críticas, nuestra posición es que permiten cuestionar la necesidad de trabajar únicamente con el lenguaje y las categorías naturales de éste, como base del estudio psicosocial del conocimiento social de las emociones. Igualmente, aceptamos que la concepción de las categorías prototípicas tiene limitaciones teóricas. Sin embargo, pensamos que ésta permite una serie de pasos de investigación del conocimiento social, sin que esto prejuzgue sobre la totalidad de la teoría (generación de jerarquías verticales mediante agrupamientos de similaridad-diferencias, generación de núcleos definitorios de conceptos mediante tareas de recuerdo y reconocimiento, establecimiento de la relación entre tipicidad y recuerdo libre y reconocimiento, contrastación del efecto reconstructivo del prototipo en la memoria, etc.). Además, como plantea Bellelli (1987), el carácter concreto y de imagen de los prototipos (de los ejemplares en este caso), permiten que los conceptos naturales se «ontologicen», es decir, que se objetiven las representaciones sociales de las emociones en casos, concretos, materiales y vivaces, que se materialicen los significados del conocimiento emocional. Los prototipos del conocimiento social no sólo clasifican, sino que con su carácter de escenario antecedente-reacción-autocontrol, orientan y prescriben las conductas. Es decir, los prototipos emocionales también jugarían un rol de anclaje de las representaciones sociales de las emociones.

En síntesis, aun reconociendo que hay elementos que permiten concebir la cognición social de las emociones como conceptos y escenarios prototípicos, compartimos las críticas realizadas en lo referente a la importancia de los modelos culturales y representaciones sociales. Las categorías o conceptos emocionales se definirían a partir de redes taxonómicas-explicativas-folklóricas y no por mera similaridad-diferencia. Pese a reconocer su interés, también hay que aceptar que la aproximación del conocimiento social de las emociones como categorías naturales prototípicas, peca del individualismo que caracteriza a la cognición social. Una forma de superar esto, es tener en cuenta cómo variables contextuales determinan, no sólo el despliegue o la narración de los fenómenos emocionales, sino también cómo afectan a la estructura y al contenido de éstos. Es lo que creemos que puede hacerse al analizar, cómo la densidad de la red social, y el rol sexual afectan el contenido y la estructura de los prototipos y categorías que los sujetos poseen sobre las emociones. 
Roles sociales, soporte social objetivo, subjetivo y construcción de los prototipos emocionales

Primero, la implicación en varios roles provee de acceso a diferentes segmentos sociales, a diferentes recursos y perspectivas cognitivas y de valores. Una mayor diversidad de roles, está potencialmente orientada a una mayor complejidad cognitiva del conocimiento emocional, a un acceso a diferentes visiones del mundo y, a diferentes experiencias internalizadas en esquemas cognitivos más complejos y diferenciados -es la clásica idea del interaccionismo simbólico, de que la riqueza del sí mismo depende de la riqueza de las interacciones, de la cantidad de roles que el sujeto juega-. Thoits (1986) ha encontrado que un mayor número de roles está asociado a menor ansiedad y depresión. Algo similar se ha encontrado en relación a las redes sociales de baja densidad, es decir de menor interconexión y que implican mayor número de roles (Hobfooll \& Stokes, 1988).

Segundo, dado que las emociones son esencialmente fenómenos interpersonales, una mayor cantidad de relaciones y contactos sociales también se puede asociar potencialmente a una mayor riqueza de experiencias afectivas. De hecho, se ha encontrado que los sujetos de mayor soporte social objetivo, es decir, de mayor contacto social, tienen más emociones positivas y menos negativas; y que el soporte actúa como factor protector ante situaciones estresantes provocadoras de emociones negativas (Paez et al., 1986).

Tercero, a una mayor densidad de roles se puede corresponder una mayor capacidad de enfrentamiento emocional. En la medida que los recursos y tácticas de enfrentamiento de las experiencias afectivas; a nivel de información, de recursos prácticos y de apoyo moral-afectivo; no son sólo variables individuales, sino que de tipo interpersonal, el acceso a una red de contactos sociales más densa y más satisfactoria subjetivamente, se asociará tendencialmente a una mayor capacidad de enfrentamiento a las experiencias afectivas. El nivel de soporte social subjetivo está más asociado que el objetivo a indicadores de síntomas ansiosos, depresivos y de malestar (Hobfooll \& Stokes, 1988). No sólo eso, sino que un sujeto con muchos contactos, pero insatisfactorios, tendrá dificultades para recibir soporte informacional, instrumental y afectivo, para validar y manejar sus emociones (Thoits, 1986; Valencia, Paez y Echebarría, 1989).

A nivel meramente cognitivo, de elaboración del significado, la interpretación y validación interpersonal de las vivencias afectivas puede jugar un rol adaptativo y enriquecedor del conocimiento sobre las emociones, de diferentes maneras. Primero, permite darle un sentido a sucesos ambivalentes y complejos. Mediante la comunicación, el individuo se ve forzado a organizar y estructurar su experiencia afectiva. El tener que utilizar el lenguaje obliga al sujeto a transformar las imágenes y vivencias difusas, en una organización temporal y secuencial. Segundo, la comunicación repetida sobre un suceso afectivo, permite revisar y analizar, a diferentes niveles, una experiencia dada, facilitando su asimilación. Un resultado claro de la investigación en cognición social, es que un suceso integrado en un esquema abstracto y congruente con él -o incongruente, pero habiendo sido elaborada su relación con el esquema - se recuerda mejor. Igualmente, un suceso procesado en mayor profundidad o a un nivel autoreferente y de significado, se recuerda mejor (Higgins \& Bargh, 1987). Tercero, la narración a otros también externaliza 
un hecho: hace que exista un referente colectivo ya sea oral o escrito, lo que finaliza el trabajo de revivir y rememorar el suceso afectivo y crea un marco colectivo de recuerdo. Por último, la discusión con otros significativos permite también reencuadrar, ver de otra manera un suceso, aumentando la flexibilidad cognitiva (Pennebaker, 1988).

Es importante señalar que, esta ausencia de narración e intercambio simbólico sobre lo afectivo, puede ocurrir por razones de personalidad (alexitímicos o sujetos con falta de lenguaje afectivo y sujetos de baja capacidad de self-disclosure o capacidad de entregar información íntima) o por las circunstancias y roles sociales que ocupan en ese momento.

\section{Roles sexuales y conocimiento social de las emociones}

Por otro lado, a nivel de roles de género, las mujeres se autoperciben y son percibidas como más emocionales (en particular emociones positivas y de vulnerabilidad son vivenciadas más intensamente por las mujeres), informan de más síntomas de emociones, son más fiables en su conocimiento social de las emociones, y entregan más información prototípica que los hombres (Scherer, Rimé \& Chip, 1989; Wood, Rhodes \& Whelan, 1989; Hendrick, 1988). De lo anteriormente expuesto, se puede postular que las mujeres: a) tienen un conocimiento social más rico de las emociones; b) son más expresivas o externalizadoras en situaciones emocionales y; c) se sentirán más afectadas o influenciadas por la activación emotiva. El hecho de que las mujeres se encuentre en roles sociales que las exponen más a experiencias con carga afectiva (roles de servicios, de cuidado de niños y ancianos, de protección emocional), así como que ellas desarrollen una «subcultura» con una reflexión y lenguaje más elaborado sobre las vivencias emotivas, explicaría estos fenómenos (Vergara y Paez, 1989). Las mujeres reciben y entregan más soporte social, así como lo valoran subjetivamente como mejor. Las diferencias se dan, en particular, sobre el soporte afectivo, ya que a nivel de información e instrumental material, las diferencias no existen. A nivel de relaciones de amistad, las mujeres pasan más tiempo compartiendo más y validando más experiencias afectivas. Además, tienen mejores puntuaciones en self-disclosure y menores en alexitimia (Hendrick 1988; Noël \& Rimé, 1988).

\section{METODO}

\section{Muestra}

La muestra era de 110 sujetos familiares y amigos no estudiantes universitarios de estudiantes de segundo y tercer ciclo de la UPV. Un $46 \%$ eran varones y un $54 \%$ mujeres. La media de edad era de 34 con un rango de 19 a 60.

\section{Instrumento}

El cuestionario constaba de tres partes:

a) La información sociodemográfica (sexo, edad, ocupación) y una lista 
de roles que el sujeto debía indicar si desempeñaba o no. Esta lista de roles cubría de manera exhaustiva los roles normalmente desempeñados en nuestro contexto (hijo, padre, pareja, tío, hermano, sobrino, abuelo, trabajador, estudiante, miembro de asociación deportiva, cultural, religiosa, de algún partido político, amigo, otros). También se le preguntaba por el número de personas con las que mantenía contacto íntimo en cada rol. Este cuestionario intentaba ofrecer un indicador de la riqueza de roles. Igualmente, se le pasaba un cuestionario de soporte social objetivo - que medía la frecuencia de contactos con compañeros de trabajo en momentos de conflicto, de favores realizados a vecinos, de visitas de amigos y personas cercanas, de personas cercanas a quien contar sus problemas personales, de participación en grupos de deportes y comunitarios, y si tenía pareja o confidente-. Este cuestionario tiene una fiabilidad mediana ( .38 en una muestra aleatoria de 300 sujetos de Navarra; García, 1986, en Barrio et al., 1989), pero, tiene validez -está asociado significativamente a indicadores de ansiedad y depresión- (véase Barrio et al., 1989). Se orientaba a tener un indicador de soporte social objetivo. Un tercer cuestionario de seis items (tres positivos y tres negativos) indagaba sobre la satisfacción subjetiva con el apoyo informacional, material y afectivo, que le daba su entorno al sujeto. Este cuestionario posee una fiabilidad alta (.70 en la muestra antes citada) y a nivel de validez se ha asociado a indicadores de síntomas depresivos y ansiosos (Barrio et al., 1990). Este instrumento buscaba un indicador de soporte social subjetivo.

Con estos elementos, se podía operacionalizar la densidad de relaciones sociales e identificar el sexo del sujeto.

b) En el cuestionario se proponían dos formatos; uno en el que se le pedía al sujeto que describiera cómo vivía un incidente emocional la mayoría de la gente (condición «observador"), y otro en el que se le pedía que describiera cómo había vivido él mismo, un incidente emocional. Se le pedía que describiera cuatro emociones; alegría, tristeza, cólera-rabia-ira y miedo. Con esta parte, se buscaba operacionalizar la perspectiva de generación de la respuesta sobre las características de las emociones. En este artículo, sólo trabajaremos con la versión en primera persona del cuestionario. Las narraciones de miedo se han mostrado bastante insensibles a las variables psicosociales, tanto en nuestra investigación como en otras (Scherer, Rimé \& Chip, 1988), por lo que las hemos excluido del análisis.

c) En la descripción de cada incidente se pedía que: A) «Describa con detalle qué sucede, qué lo causa o lo provoca»; B) 1) «Qué sintió o pensó» (o qué siente y piensa la mayoría de la gente), 2) «Qué se dicen a sí mismo» (o qué se dijo a sí mismo), 3) «Qué señales físicas sienten» (o sintió); 4) «Qué hacen o cómo actúan» (o qué hizo y cómo actuó), 5) "Cuánto duró el incidente», y 6) «Cómo se resolvió». Estas instrucciones se replicaron de la investigación de Shaver et al. (1987). Había cuatro órdenes aleatorios de presentación de las emociones, que se utilizaron para controlar efectos de presentación. Después de que el sujeto respondiera al conjunto de preguntas abiertas antes descritas; se le pidió que él mismo categorizara sus respuestas abiertas con los atributos prototípicos de las cuatro emociones. Estas respuestas se consideran como manifestaciones de recuerdo libre de la memoria emocional. Por último, se les pidió que «marcaran con un círculo las respuestas con las cuales está Ud. más de acuerdo que pertenecen a la experiencia típica» de la emoción en cuestión. Esto se consideró como manifestación del recono- 
cimiento del conocimiento emocional. El prototipo de cada emoción se construyó a partir de los atributos encontrados por Hazan \& Shaver en al menos el $10 \%$ de su muestra de 120 estudiantes de USA, agregándosele los atributos encontrados por Scherer et al. en su investigación transcultural de las emociones que fueron mencionados por al menos el $10 \%$ de la muestra total y que no se reproducían en el prototipo de Hazan \& Shaver. Este prototipo se ha validado a nivel de la tipicalidad de los atributos, de la fiabilidad y validez de la categorización de respuestas y de la relación entre tipicalidad y recuerdo (Paez y Vergara, 1990).

\section{Procedimiento}

Los cuestionarios se pasaban en la casa-habitación del sujeto por un estudiante de segundo o tercer ciclo como parte del trabajo de un curso de Psicología. Estos últimos habían contestado ellos mismos los cuestionarios previamente y habían sido iniciados sobre la información que había que obtener. A los sujetos se les hizo autocategorizar, con la ayuda de los entrevistadores, sus narraciones abiertas.

\section{RESULTADOS}

Como una primera forma de contrastar las asociaciones entre relaciones sociales y atributos prototípicos de las emociones, agrupamos todos los atributos recordados y categorizados (narrados por el sujeto y categorizados $=1$; ausencia $=0$ ) por áreas (reacciones abiertas interpersonales, expresivas, físicas, mentales o subjetivas y de autocontrol). También hicimos lo mismo con el reconocimiento de un atributo como típico (si lo juzga como típico de la emoción revisando la lista de atributos $=1$, si no=0). La Tabla Ia presenta los resultados.

Como se puede ver, hay asociaciones significativas entre el sexo y casi todas las áreas, reafirmando que las mujeres recuerdan más en general, expresan, actúan y vivencian más la emoción, así como también la autocontrolan más. El número total de roles (totalrol) está también asociado a mayor número de reacciones interpersonales, más reacciones subjetivas y en general -salvo con autocontrol-, se constata que todas las asociaciones son similares y se aproximan a la tendencialidad. El soporte subjetivo no tiene asociaciones fuertes, salvo con autocontrol (a mayor insatisfacción, más autocontrol) y lo mismo ocurre con el soporte objetivo, que se asocia negativamente sólo con reacciones físicas. Examinando La Tabla Ib - vemos que la relación con el reconocimiento de atributos replica de manera más clara los resultados anteriores - en particular, el soporte objetivo aparece asociado significativamente a un menor reconocimiento de una serie de atributos. Por otro lado, vemos que la posibilidad de que las asociaciones entre relaciones sociales se deban a la covarianza entre éstas y factores como la edad y el sexo, es baja. Si bien la edad tiene una asociación positiva con el recuerdo y el reconocimiento de los atributos emocionales, la asociación entre ésta y las variables de relaciones sociales, no es muy alta. Además, si bien las mujeres tienden a tener más soporte objetivo y menor subjetivo, las asociaciones son bajas. De 
TABl.A I

Ia) Correlaciones entre relaciones sociales y recuerdo libre categorizado

\begin{tabular}{lcccccc}
\hline & TOAI & TOFIS & TOEX & AUTCON & TOPSI & TOREAC \\
\hline EDAD & $.2146 *$ & $.1699 \&$ & $.1749 \&$ & -.0124 & .1198 & $.2019^{*}$ \\
SEXO & .1591 & $.2665^{*}$ & $.2402^{*}$ & $.1643 \&$ & $.2303 \%$ & $.2719^{*}$ \\
SOPREAL & -.0269 & -.1260 & .0049 & -.0379 & -.1050 & -.0706 \\
SOPEMO & .0916 & -.0264 & .0008 & .1256 & -.0815 & .0131 \\
TOTALROL & $.1802 \&$ & .1368 & .1591 & .0446 & $.1804 \&$ & $.1943^{*}$ \\
STR1 & .0721 & .0191 & .1024 & -.0287 & .0738 & .0768 \\
& $\mathrm{~N}=108$ & $\&-.10$ & & $*-.05$ & $*$ & \\
\hline
\end{tabular}

Ib) Correlaciones entre relaciones sociales y acuerdo con el carácter típico del atributo

\begin{tabular}{lcccccc}
\hline & TOAI & TOFIS & TOEX & AUTCON & TOPSI & TOREAC \\
\hline EDAD & $.1932 \&$ & .1251 & .0969 & $.1481 \&$ & $.1665 \&$ & $1769 \&$ \\
SEXO & .1042 & $.2261 * *$ & .1158 & $.1970^{*}$ & .1405 & $.1835 \&$ \\
SOPREAL & -.1245 & -.2095 & -.0761 & -.1263 & $-.1901 \&$ & $-.1767 \&$ \\
SOPEMO & .0565 & -.0627 & .0495 & .1026 & -.0348 & .0166 \\
TOTALROL & $.2103^{*}$ & $.1776 \&$ & $.1912 \&$ & .0949 & .17198 & $.2258 * *$ \\
STR1 & .0433 & .0056 & .0616 & -.0428 & .0058 & .0334 \\
& $\mathrm{~N}=108$ & $\&-.10$ & & $*-.05$ & $* \%-.01$ & \\
\hline
\end{tabular}

Ic) Correlaciones entre relaciones sociales, edad y sexo

\begin{tabular}{lcrrrr}
\hline & EDAD & SEXO & SOPREAL & SOPEMO & TOTALROL \\
\hline EDAD & & & & & \\
SEXO & .0192 & & & & \\
SOPREAL & -.0304 & .1295 & & & \\
SOPEMO & $.2050 *$ & -.1112 & -.1065 & & $.7469 * *$ \\
TOTALROL & $.1609 \&$ & -.0373 & $.1895 \&$ & -.0207 & \\
STR1 & .0270 & .0833 & $.7366^{* *}$ & $-.3509 * *$ & .01 \\
& $\mathrm{~N}=108$ & $\&-.10$ & $*-.05$ & $* \%-.01$ \\
\hline
\end{tabular}

Id) Coeficientes beta de la regresión múltiple entre sexo, edad, relaciones sociales $y$ acuerdo con el carácter típico del atributo

\begin{tabular}{|c|c|c|c|c|c|c|}
\hline & TOAI & TOFIS & TOEX & AUTCON & TOPSI & TOREAC \\
\hline EDAD & .13 & .08 & .04 & .09 & .13 & .12 \\
\hline SEXO & .15 & $.27^{* * *}$ & .15 & $.24 \%$ & $.18 \&$ & $.22 *$ \\
\hline SOPREAL & $-.20 \%$ & $-.53^{\circ \%}$ & -.13 & $-.17 \&$ & $-.26^{* * \%}$ & $-.25 \%$ \\
\hline SOPEMO & .02 & -.09 & .04 & .09 & -.07 & .00 \\
\hline TOTALROL & $.24 \%$ & $.24 \%$ & $.21 \%$ & .12 & $.21 *$ & $.26^{* *}$ \\
\hline $\mathrm{R}$ & .34 & .43 & .27 & .32 & .37 & .41 \\
\hline R cuadrado & .12 & .19 & .07 & .10 & .14 & .17 \\
\hline \multicolumn{2}{|c|}{$\mathbf{N}=108$} & $\&-.10$ & & $*-.05$ & $\because \because-.0$ & \\
\hline
\end{tabular}

Notas: $\quad$ TOAI $=$ Total Reacciones Interpersonales.

TOFIS $=$ Total Reacciones Físicas.

TOEX $=$ Total Reacciones Expresivas.

TOPSI = Total Reacciones Subjetivas o "Mentales".

TOEX $=$ Total Reacciones Expresivas.

TOREAC $=$ Total General de Atributos.

Para la clasificación de atributos véase Paez y Vergara 1990 y la Tabla II.

SOPREAL = Soporte Objetivo.

SOPEMO = Soporte Subjetivo.

TOTALROL $=$ Número Total de Roles.

STRY1 = Puntuación Factorial de los tres anteriores. 
todas maneras, las regresiones múltiples llevadas a cabo sobre el total de atributos emocionales, confirman que el número de roles y el sexo tienen asociaciones positivas con el reconocimiento de atributos prototípicos de las emociones, mientras que el mayor contacto social objetivo tiene una asociación negativa - los coeficientes beta significativos indican que estas asociaciones son específicas y no se deben a las asociaciones entre las variables antes mencionadas ni a las de éstas con la edad - (véase la Tabla Id). Vemos ahora a nivel de asociaciones bivariadas cuáles son las asociaciones significativas.

La Tabla II, muestra las correlaciones puntobiseriales entre el reconocimiento de si un rasgo es típico o no de una emoción (primera línea; si el sujeto reconoció como típico $=1, \mathrm{No}=0$ ), y del recuerdo libre categorizado (segunda línea; si el sujeto recordó libremente y se categorizó su respuesta $=1$, si no $=0$ ) con las medidas de soporte social objetivo (columna uno; a mayor puntuación mayor soporte), satisfacción subjetiva con el soporte (segunda columna; a menor puntuación, más satisfacción con el soporte) y de número de roles (tercera columna; a mayor puntuación, más roles), así como la puntuación factorial (varimax, un factor impuesto, método AR) de los tres indicadores de relaciones sociales antes descrito - cuarta columna-. La quinta columna, presenta las correlaciones phi entre sexo y atributos (hombre $=0$, mujer $=1$ ).

Las asociaciones replicadas en las tareas de recuerdo y reconocimiento entre atributos prototípicos de emociones y el soporte social objetivo, subjetivo y el número de roles, son de 7,11 y 17 respectivamente $(8 \%, 13 \%$ y $20 \%$ de diferencias tendenciales o significativas replicadas en las dos tareas cognitivas - como punto de comparación el total de diferencias replicadas a nivel del sexo es del $21 \%$, como se expondrá más adelante).

Si revisamos la influencia de las relaciones sociales en la construcción del conocimiento de las emociones, constatamos que tanto en la tarea de recuerdo y narración, como en la tarea de reconocer el carácter típico en su experiencia emocional de un atributo emocional, un mayor número de roles está asociado regularmente a un mayor recuerdo y tipicidad de ciertos antecedentes - recibir estima y sensaciones agradables en alegría; pérdida de poder, estatus y planes . no marchan en cólera; empatizar y situación de impotencia en tristeza-. Igualmente, un mayor número de roles se asocia a una mayor reacción física - sentirse más enérgico en alegría; sentir más calor en cólera y sentirse más cansado en tristeza-. Con respecto a las reacciones interpersonales, a mayor número de roles, mayor recuerdo y tipicidad atribuida a ser cortés, no apartarse de la gente, hacer cosas agradables y compartir sentimientos en alegría - no hay asociaciones en tristeza y sólo a nivel de reconocimiento, con mayor actividad interpersonal en cólera - ataque verbal causa de la cólera. A nivel expresivo, el mayor número de roles está asociado a más gestualidad; no sonreír, fruncir el ceño, en cólera, y a no sonreír en tristeza. Además, a nivel del reconocimiento hay una asociación con sonreír en la emoción de alegría. Por último, a mayor número de roles, más se recuerda y evalúa como típico, el adoptar una actitud positiva en la emoción de alegría. En su conjunto, creemos que está claro que a mayor variedad de perspectivas y de roles, más rico el conocimiento social de las tres emociones examinadas.

Si tomamos ahora la dimensión de soporte social objetivo o frecuencia de contactos sociales, vemos que el tipo de influencia no se solapa con el anterior. Los sujetos de mayor contacto social son menos sensibles a ciertos 
TABLA II

Primera línea: Típico

Segunda línea: Recordó libremente en la narración

PROTOTIPO DE ALEGRIA $S I=1 ; N_{0}=0$

\begin{tabular}{|c|c|c|c|c|c|}
\hline ANTECEDENTES & 1 & 2 & 3 & 4 & 5 \\
\hline ale01 Tarea exito & $.21^{*}$ & & $.18 *$ & $.21^{*}$ & -.13 \\
\hline ale02 Obtener deseaba & -.14 & & & & $.24 \%$ \\
\hline ale03 Recibir estima & & .15 & $\begin{array}{l}.25^{* *} \\
.25^{* \%}\end{array}$ & $\begin{array}{l}.16^{*} \\
.17^{*}\end{array}$ & $\begin{array}{l}.13 \\
.14\end{array}$ \\
\hline ale04 Obtener algo & & & & .15 & \\
\hline ale05 Recibir sorpresa & & & .13 & & \\
\hline ale07 Sensac. agradables & & & $\begin{array}{l}.18^{*} \\
.23^{*}\end{array}$ & .16 & \\
\hline ale08 Ser aceptado & & & & & \\
\hline ale09 Recibir amor & & $\begin{array}{l}-.14 \\
-.16\end{array}$ & $.18^{*}$ & $\begin{array}{l}.20^{*} \\
.17^{*}\end{array}$ & $\begin{array}{l}.18^{*} \\
.22^{*}\end{array}$ \\
\hline ale06 Realidad excede expectat. & $\begin{array}{l}-.15 \\
-.16\end{array}$ & $\begin{array}{l}-.23 * \% \\
-.16\end{array}$ & & $-.23 \%$ & \\
\hline RESPUESTAS FISICAS & 1 & 2 & 3 & 4 & 5 \\
\hline ale18 Excitado emoc. & & & & & .16 \\
\hline ale19 Enérgico & & $-.19^{*}$ & $\begin{array}{l}.22 \% \\
.20^{*}\end{array}$ & $\begin{array}{l}.17^{*} \\
.16^{*}\end{array}$ & $\begin{array}{l}.39 \% \\
.40^{*} \%\end{array}$ \\
\hline ale20 Brincar bailar & $.16^{*}$ & & & $.16 *$ & $.22^{*}$ \\
\hline ale21 Dar saltos & .14 & & & & \\
\hline ale28 Relaj. muscular \& & -.14 & & & & \\
\hline $\begin{array}{l}\text { REACCIONES ABIERTAS } \\
\text { INTERPERSONALES }\end{array}$ & 1 & 2 & 3 & 4 & 5 \\
\hline ale10 Cortés, amistoso & & $.16^{*}$ & $\begin{array}{l}.27 * * \\
.24 *\end{array}$ & & \\
\hline ale14 No apartarse gte. & & & $\begin{array}{l}.15 \\
.14\end{array}$ & & \\
\hline ale 11 Hacer cos. agr. otr. & & & $\begin{array}{l}.27 * * \\
.22 *\end{array}$ & $\begin{array}{l}.16 * \\
.14\end{array}$ & \\
\hline ale13 Compartir sentim. & & & $\begin{array}{l}.29 * \% \\
.25 \%\end{array}$ & $.19 *$ & .14 \\
\hline
\end{tabular}


REACCIONES ABIERTAS

EXPRESIVAS

1

23

4

5

ale17 Risa

ale25 Sonreír

$-.13$

a 25 Sonreir

REACCIONES MENTALES

DE PENSAM. SENTIM.

$\begin{array}{lllll}1 & 2 & 3 & 4 & 5 \\ .13 & & .25^{* *} & & .22^{* *} \\ & .18^{*} & .13 & & .21^{*}\end{array}$

ala15 Actitud positiva

$\begin{array}{lll}.16^{*} & .14 \quad .24 * \% \\ & & .27 \%\end{array}$

ale16 Umbral alto preoc.

Total atributos de alegría $=28$

PROTOTIPO DE TRISTEZA

$\begin{array}{lccccc}\text { ANTECEDENTES } & 1 & 2 & 3 & 4 & 5 \\ \text { Tris01 Resultado indeseable } & -.15 & & .15 & & -.14\end{array}$

Tris02 Muerte ser querido

$-.16$

Tris04 Rechazo, desaprobación

$.13 \quad .18^{* *}$

Tris07 Impotente, inacapaz

$\begin{array}{ll}.30 * & .26 * * \\ .18 * & .13\end{array}$

Tris08 Empatizar

$.21 * \quad .14$

$.25 \% \quad .18 *$

Tris06 Cosas van peor

REACCIONES FISICAS

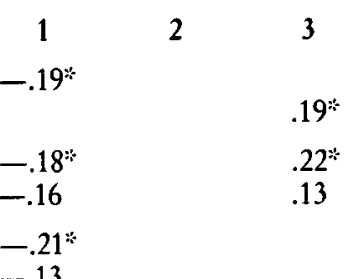

4

5

Tris09 Inactivo, apático

Tris10 Cansado

$22 *$

Tris11 Movim. lentos

$-.13$

$-.17^{*}$

$-.14$

Tris12 Postura decaída

REACCIONES ABIERTAS

EXPRESIVAS

1

2

3

5

Tris16 Decir cosas tristes

Triș18 Llorar

Tris17 No sonreír, ceño

$-.19 *$

$-.21^{*}$ 
REACCIONES MENTALES

DE SENTIMIENTO

Y PENSAMIENTO

Tris19 Irritable

1

$\begin{array}{llll}2 & 3 & 4 & 5\end{array}$

.13

$.21 *$

Tris20 Melancólico

$-.24 \%$

.17

$.18^{*} \quad .19^{*}$

Tris21 Visión negativa

Tris22 Rendirse

$-.16$

Tris23 Autocrítica

$-.18^{*}$

$-.13$

Total atributos de tristeza $=26$

PROTOTIPO DÉ COLERA

$\begin{array}{llllll}\text { ANTECEDENTES } & 1 & 2 & 3 & 4 & 5 \\ \begin{array}{l}\text { Co101 Predisposición por exp. } \\ \text { previas }\end{array} & & & & & .21^{*} \\ .17^{*}\end{array}$

Co102 Pert. poder

$\begin{array}{lll}-.16^{*} & .15 & .18^{*} \\ & .17^{*} & .17^{*} \\ & .17^{*} \\ & .17^{*}\end{array}$

Co105 Dolor fís. o temido

$\begin{array}{lll}.14 & -.13 \quad .14\end{array}$

Co106 Injusticia

$-.16$

RESPUESTAS FISICAS

12

Col22 Rubor

2

3

5

$-.20^{*}$
$-.20^{*}$

$-.15$

.16

Col28 Calor \&

$-.29 \%$

$-.20^{*}$

$-.13$

Col29 Síntomas musculares \&

RESPUESTAS ABIERTAS

INTERPERSONALES

1

2

3

5

Col14 Ataque físico causa cólera

$-.23 \%$

$-.21 *$

Col13 Ataque físico a otro objeto

Col15 Descontrol, cond. emoc. ftes $-.23 \%$

Col19 Com. no verbal. des

Col25 Abandono cont. soc.

Col08 Ataque verbal causa cólera $\quad-.15$ 


\section{8}

RESPUESTAS ABIERTAS

EXPRESIVAS

1

2

3

4

5

Col23 Llorar

.14

$.33 \%$

$.29 * \%$

Col09 Chillar

.16

$.21 \%$

Col20 Discutir

$.25 \%$

$.27 * \%$

Col07 Blasfemar

Col11 Apretar puños

Col12 Gestos agresivos $.18^{*}$

Col20 No sonreír, fr. ceño

.17

$.20 \%$ $.18^{*-5}$

$.18^{*} \quad .24 \%$

$.15 \quad .27 \%$

Col17 Pisar fte. andar

.14

.14

Col21 Mostrar, rechinar dientes

REACCIONES MEN'TALES

DE PENSAM. SENTIM.

Col16 Imaginar atacas

12

3

4

5

$-.18^{*}-.20^{*}$

$-.27^{* *}$

Col26 Pensar estar cierto

$-.23 \%$

$-.20^{*}$

AUTOCONTROL

1

3

4

5

.14

$.23 \%$

.14

Total atributos de cólera $=31$

Columna 1 = Soporte objetivo

Columna 2 = Soporte subjetivo

Columna $3=$ Numero de roles

Columna $4=$ Puntuación factorial

Columna $5=$ Sexo $1=$ hombre, $2=$ mujer

Sólo se presentan los atributos prototípicos que han tenido asociaciones significativas las correlaciones sin asterisco son tendenciales, $*=$ menor que $.05 \mathrm{y} *=$ menor que .01 .

antecedentes, como por ejemplo, que la realidad excede las expectativas en alegría. A nivel de reconocimiento, son menos sensibles a antecedentes como pérdida de poder en cólera, obtener lo que se deseaba en alegría y, resultado indeseable en tristeza. A nivel de recuerdo, asocian más tarea de éxito en alegría, menos muerte en tristeza, y menos pérdida de poder en cólera. Parece como si el soporte social objetivo protegiera a los sujetos, de ciertos antecedentes de emociones negativas o en su defecto, que ciertos antecedentes serían menos típicos porque la realidad colma sus expectativas -asociación negativa con realidad excede expectativas, en alegría y, positiva con tarea 
de éxito-. Igualmente, a mayor contacto social, menor intensidad de la reacción física emocional; menor recuerdo y tipicalidad de rubor y calor en enojo; menos cansado y movimientos lentos en tristeza. También hay una asociación negativa con la expresividad; no sonreír en tristeza, y con la interacción; menos conductas emocionales fuertes en enojo. Si tomamos los resultados de reconocimiento, vemos que el perfil es similar; menor sensibilidad a la pérdida de poder como antecedente de cólera, al igual que asociación negativa con síntomas físicos, con chillar y con ataque verbal, y con reacciones psicológicas, como imaginar que atacas y pensar que estás en lo cierto, y autocrítica en tristeza - la asociación con la expresividad, es también negativa con no sonreír en tristeza-. El hecho de que la relación con la expresividad sea positiva en alegría - brincar, en recuerdo, y dar saltos, en reconocimientoindica que estamos en presencia de un fenómeno de monitoraje o de autocontrol de la expresividad y no únicamente de inhibición de las reacciones. La asociación con el efecto psicológico de la alegría - a nivel del reconocimientoes positiva, aunque pequeña, reafirmando lo anterior.

En lo referente a las asociaciones entre la satisfacción subjetiva con el soporte social y los atributos de los prototipos emocionales, encontramos que a mayor satisfacción, más recuerdo y reconocimiento de ciertos antecedentes de la alegría (recibir amor y realidad excede a las expectativas), más expresión y reacciones subjetivas en tristeza (llorar, estar melancólico, rendirse) y cólera (síntomas musculares y ataque físico causa cólera). Las asociaciones positivas (en este caso con la insatisfacción con el soporte) se dan con ciertos antecedentes de cólera (pérdida de poder, status), expresiones (fruncir el ceño, no sonreír), interpersonales (abandono del contacto social) e internas (redefinir situación).

Con respecto a las diferencias de género, se confirma en 18 comparaciones que las mujeres son más sensibles a ciertos antecedentes sociales (recibir estima y amor en alegría y predisposición a enojarse por experiencias anteriores estresantes en cólera) de las emociones, pero, sobre todo se confirma que muestran más síntomas físicos (enérgico en alegría, postura decaída en tristeza), más rasgos expresivos (sonreír en alegría, llorar en tristeza y cólera, más pisar fuerte en cólera), más acciones interpersonales como chillar, discutir y conductas emocionales fuertes en cólera, y más influencia de la afectividad en lo subjetivo (visión positiva en alegría, irritable, melancólica y autocrítica en tristeza) aunque también mayor autocontrol (redefinir la situación en cólera). La única superioridad masculina, se da en síntomas musculares en cólera.

\section{TABLA III}

Coeficientes Beta de la regresión múltiple entre las variables de relación social, el sexo y la suma de atributos en que se solapaban influencias significativas

\begin{tabular}{lcccc}
\hline & SEXO & SOPREAL & SOPEMO & TOTALROL \\
\hline Total General & $.43^{* *}$ & -.17 & -.04 & $.32^{* *}$ \\
Total Alegría & $.35^{* *}$ & -.05 & .01 & $.38^{* *}$ \\
Total Cólera & $.37^{* *}$ & $-.22^{*}$ & .05 & .13 \\
Total Tristeza & $.29^{*}$ & $-.19^{*}$ & $-.23^{*}$ & $.19^{*}$ \\
\hline
\end{tabular}

Comparando la influencia simultánea de las variables anteriores sobre la suma total de resultados en los que habían resultado significativos que se solapaban, constatamos que el sexo sigue influyendo específicamente en el 
reconocimiento de estos atributos, que a continuación influye el número de roles, en tercer plano el contacto social y por último el soporte subjetivo. Este último, sin embargo, tiene una influencia específica en la emoción de tristeza, de facilitación del reconocimiento - a mejor soporte subjetivo, más reconocimiento de ciertos atributos-. El soporte objetivo, tiene un papel inhibidor del reconocimiento en tristeza y cólera, y el número de roles un papel facilitador en tristeza y alegría.

Con el fin de contrastar la asociación entre las relaciones sociales, el sexo y los escenarios típicos, procedimos a realizar un análisis de correspondencias sobre los atributos recordados de cada emoción. Los atributos que pesaban en un factor y que tenían una correlación con él superior a .30 se adicionaron, para tener una puntuación total que representara a un escenario. Dada las limitaciones de la muestra y de la tarea (recuerdo de un episodio y no de todos los tipos posibles), no se trataba tanto de extraer una tipología de escenarios representativa de una cultura dada, como de constatar que los escenarios típicos también se asociaban a las variables de relaciones sociales y al sexo.

El soporte objetivo, está asociado positivamente al escenario de alegría de éxito concreto y excitación, al escenario de tristeza de problemas y llorar, y negativamente al escenario de alegría de aceptación, relajación y aproximación social, y al de tristeza de fracaso y bajón físico. El total de roles está asociado positivamente a los escenarios de alegría de éxito concreto y excitación, al de recibir estima, aproximarse y comunicar con otros, y al escenario de tristeza de problema y tristeza menor. El número de roles reproduce la accesibilidad de escenarios moderados de tristeza y alegría, y sobre todo se asocia al escenario de antecedentes sociales y aumento del contacto interpersonal. Lo más sorprendente es la gran cantidad de resultados asociados al soporte subjetivo (28\% de contrastes significativos versus $12 \%$ para el número de roles y $16 \%$ para el soporte objetivo; asociado a las diferencias sexuales hay un $32 \%$ de contrastes). Los escenarios asociados a un soporte social subjetivo positivo son los de realidad positiva y comunicación con otros en alegría, los de pérdida, melancolía y autocontrol y el de problemas y llorar en tristeza, y el de fracaso y agresión gestual y directa en cólera. Estos cuatro escenarios más accesibles para los sujetos de buen soporte subjetivo, se sitúan en un eje de mayor expresión, mayor actuación interpersonal y mejor manejo de la emoción; reafirmando las conclusiones extraídas de los análisis bivariados. La insatisfacción subjetiva con el soporte, está asociada a problemas y tristeza menor en tristeza, así como a los escenarios de antecedentes sociales - estima y aceptación-y de aumento del contacto social - sugiriendo que estos hechos son más salientes por su baja frecuencia-. A nivel de sexo, los hombres tienen más accesibles los escenarios de fracaso y agresión gestual, así como los de falta de respeto y ataque en cólera, mientras que las mujeres tienen más accesibles los escenarios de problemas y enojo menor, así como los de predisposición y expresión; confirmando la mayor expresividad y menor asertividad de las mujeres en las situaciones de enojo. Igualmente, la mayor sensibilidad de las mujeres a los antecedentes sociales de las emociones y a sus afectos subjetivos, se confirma por el hecho de la asociación positiva entre sexo y los escenarios de recibir estima y aproximarse, comunicar y con el de recibir estima y sentirse enérgico fsica y subjetivamente en alegría. En el mismo sentido, se puede interpretar la 
TABLA IV

Correlaciones entre relaciones sociales y la suma de atributos asociados entre si por el análisis factorial

\section{SOPREAL SOPEMO TOTALROL STRY1 SEXO EDAD}

Escenarios de alegría

SAL1 CAUSA LOGRO-

EXCITACION

Ant-obtener lo que se deseaba; $.19 \% \quad .08$

$.23^{3 *}$

$.24^{* *}$

$.11 \quad-.04$

tarea éxito logro;

sorpresa agradable.

Reac-brincar-sentirse excitado.

SAL2 ACEPTACION.

RELAJ.-APROXI.

.148

$.148 \quad-\infty$

$-.13 \quad-.05$

.06

Ant-ser aceptado, correspondido.

Reac-relajación muscular;

acercarse gente;

ser cortés, amistoso;

umbral alto preocupación;

hacer cosas agradables para otros

SAL5 ESTIMA-APROX. Y
COMUNICAR
.07
Ant-recibir estima.
Reac-hacer cosas agradables otros;
ser cortés;
decir cosas positivas;
compartir sentimientos;
actitud positiva, fijarse lado bueno;
hablar mucho, estar comunicativo

$.18^{*}$

$.23^{* *}$

$.14 \&$

.00

.22

\section{SAL7 ESTIM «-ENERGICO}

FIS. Y PS.

.$\infty \quad 10$

.10

.04

$.41^{*} \quad .26^{* \%}$

Ant-rec. estima.

Reac-umbral alto para preocupación, enojo;

enérgico;

act. positiva

SAL 8 REAL. POS. Y

COMUNICACION

$-.04-.18^{*}$

.00

$.03-.11$

.01

Ant-obtener lo deseaba;

recibir estima;

realidad excede expect.

Reac-umbral alto preoc.;

voz entusiasta;

hablar mucho, estar comunicativo

\section{Escenarios de tristeza}

STRI1 FRACASO Y BAJA

FISICA
Ant-no conseguir quería.

$-.14 \& \quad-.02$

.04

$-.05 \quad-.02$

.08

Reac-inactivo;

cansado;

mov. lentos

hablar poco 
STRI2 FRACASO Y TRIS.

MELANC.

.04

.12

.04

.02

$.21^{*}$

$.17^{*}$

Ant-resultado negativo.

Reac-melancólico;

expresar cosas tristes;

visión negativa;

postura decaída;

irritable

STRI3 RECHAZO PLAN

MAL ABANDONO

CONTACTO Y

AUTOCRITICO

$.07 \quad-.12$

.10

$.14 \&$

$.07-.12$

Ant-rechazo, desaprobación;

cosas van peor esperado.

Reac-inactivo;

hablar poco;

abandono contacto social;

melancólico;

autocrítica

STRI5 PERDIDA,

MELANC. Y AUTO CON-

TROL

$-.13-.20$

.00

$-.02$

.168

.04

pérdida relación.

Reac-hacerse activo;

melancólico;

autocrítica

STRI6 PROBLEMA Y

TRIS.MENOR

$-.08-168$

$.18^{*}$

$.02 \quad-.02$

.05

descubrirse impotente;

cosas van peor esperado.

Reac-no sonreír, fruncir ceño

STRI9 PROBLEMA Y

$\begin{array}{lllllll}\text { LLORAR } & .16 \& & -.17^{*} & -.08 & .09 & .05 & -.12\end{array}$

Ant-cosas van peor esperado.

Reac-llorar

Escenarios de cólera

SCO3 ENOJO MENOR Y

EXPRESION MOTORICA

Y VERBAL

$.00 \quad-.03$

.09

$.06 \quad .15 \& \quad-.02$

Ant-predisposición a enojarse

por experiencias previas o por estrés, fatiga;

Dolor físico o psíquico temido o real.

Reac-blasfemar;

ataque verbal;

cerrar puños;

rechinar, enseñar dientes.

SCO4 FRACASO

Y AGRESION GESTUAL

Y DIRECTA

$.06 \quad-21 \%$

.10

$.15 \& \quad-.19 *-.04$

Ant-cosas no marchan como se planeó.

Reac-blasfemar;

centrar atención en la situación sin atender a nada más;

gestos amenazadores;

atacar causa del enojo;

atacar a otra cosa que la causa del enojo. 
SCO5 PREDISPOSICION

Y EXPRESION

MOTORICA Y VERBAL

Ant-predisposición.

$\begin{array}{lllll}.07 & .10 & -.03 & -.01 & .03\end{array}$

Reac-blasfemar;

chillar;

cerrar puños

pisar fuerte al andar;

rechinar, enseñar dientes

SCO6 FALTA RESPETO,

NERVIOSISMO Y

REACC. MOTORICA

Ant-pérdida repentina de poder, status, respeto.

.09

$-.01 \quad-.22 *-.12$

Reacc-cerrar puños;

nerviosismo;

centrar atención;

síntomas musculares

NOTAS: Sólo se reproducen los escenarios asociados a alguna variable estudiada. En cada escenario se reproduce el número del factor $(\mathrm{SC}) 6=$ factor número 6 del análisis de correspondencias múltiple de atributos de cólera) y los atributos más asociados a él (Ant = antecedente, pérdida repentina de poder o status. Reac = reacciones, cerrar puños, nerviosismo, etc.). El total de factores fue de 9 para alegría, de 7 para cólera y de 9 para tristeza. Los atributos asociados por el análisis factorial y que no se han asociado a ninguna variable son los siguientes:

\section{Cólera}

SCO2: Situación errónea o injusta como antecedente; rigidez corporal; pensar que se está en lo cierto; sentir calor y ruborizarse.

SCO7: Situación errónea o injusta; predisposición por experiencias anteriores como antecedentes; abandono del contacto social; imaginar que atacas; y pensar que se está en lo cierto.

Alegría

SAL3: Sorpresa; y sensaciones agradables como antecedentes. Risa; sonreír; y hablar mucho.

SAL4: Tarea de éxito logro, recibir estima; amor, afecto; ser aceptado, correspondido como antecedentes. Comunicar buenos sentimientos; hacer cosas agradables a otros.

SAL6: Comunicar buenos sentimientos; calor; dar saltos; voz entusiasta; brincar; sentirse excitado.

SAL9: Risa; brillo en la cara; brincar.

\section{Tristeza}

STRI4: Empatizar con alguien triste como antecedente. Expresar cosas tristes; comunicar cosas tristes; hacerse activo (como autocontrol).

STRI7: Pérdida de relación, separación como antecedente. Cansado, bajo en energía; no sonreír, fruncir el ceño; hablar en voz baja.

STRI8: Rendirse; autocríticas; suprimir sentimientos negativos.

Se puede constatar que hay factores que son asociaciones entre reacciones (dos de excitación motóricagestual en alegría; una de reacciones subjetivas y autocontrol en tristeza) y otros que representan escenarios prototípicos generales no influidos por el contexto social de los sujetos.

mayor accesibilidad para las mujeres del escenario de fracaso y melancolía autocrítica en tristeza.

\section{DISCUSION}

En su conjunto, los resultados confirman que los sujetos de sexo femenino y los sujetos de mayor densidad de relaciones sociales son más sensibles a ciertos antecedentes de las emociones, que las vivencian más intensamente a nivel expresivo y de relaciones interpersonales, así como que se sienten más 


\section{4}

influidos psicológicamente por esta emoción. El mayor número de roles se asocia a una mayor actuación emocional, así como a una mayor capacidad de tomar el rol del otro (empatizar como antecedente en tristeza, mayor tendencia a comunicar) y una mayor sensibilidad a las rupturas de expectativas de rol como antecedentes de las emociones (pérdida de poder, planes no marchan en cólera, por ejemplo). El soporte social objetivo y subjetivo protegen de ciertos antecedentes negativos, favorecen la accesibilidad de otros positivos - sobre todo el primero, que se asocia además a una menor intensidad de síntomas e inferencialmente a un mayor monitoraje o autocontrol de la representación pública-. Un buen soporte social subjetivo favorece el trabajo emocional interno, subjetivo. Su déficit, también se asocia a una mayor dificultad de manejo de las emociones y a un trabajo más individual con ellas (abandono del contacto y redefinición de la situación en cólera). De esta manera, vemos que cada dimensión de las relaciones sociales influencia y aporta elementos diferentes en la construcción del conocimiento emocional prototípico. Esta influencia también se da a nivel de los escenarios o atributos de antecedentes-reacciones-autocontrol que se asocian entre sí. Particularmente relevante a este nivel, son las influencias del soporte subjetivo, cuyo déficit se asocia a la salicencia de ciertos escenarios de refuerzo social en alegría y de fracasos en tristeza. Por el contrario, la satisfacción con el soporte está asociado a escenarios de mayor despliegue social y manejo de las emociones. Por último, se reencuentra, a nivel de escenarios, la asociación entre sexo femenino y escenarios de mayor expresividad, influencia de la afectividad en lo subjetivo y mayor sensibilidad a antecedentes sociales de las emociones; mientras los hombres se asocian más a escenarios de ruptura de normas sociales y de defensa asertiva de éstas. En otros términos, no sólo los atributos, sino que también los escenarios prototípicos de las emociones, tal como se pueden inferir del conocimiento social de éstas, están influenciados por las relaciones sociales y el rol sexual de las personas.

\section{Referencias}

Averill, J. (1982). Anger and Agression. Nueva York. Springer Verlag.

Barrio, F. et al. (1989). Alienación y Afectividad. En Echebarría, A. y Paez, D. Emociones: Perspectivas Psicosociales. Madrid. Fundamentos.

Barsalou, L. y Medin, D. (1986). Concepts: Static definitions of context-dependent representations. European Bulletín of Cognitive Psychology. 6, 187-202.

Bellell.1, G. (1987). Prototypes et Representations Sociales de la Maladie Mentale. En G. Bellelli (Ed.). La Representation Sociale de la Maladie Mentale. Napoli. Liguori.

Ciorl, G. y. Ortony, C. (1988). The Semantics of the Affective Lexicon. En V. Hamilton, G. Bower y N. Frijda. Cognitive Perspectives on Emotion and Motivation. Dordrecht. Kluwer Academic Press.

Chaplin, W. et al. (1988). Conceptions of States and Traits: Dimensional Attributes with Ideals and Prototypes. Journal of Personality and Social Psychology. 54, 541-557.

Di Giacomo, J. P. (1987). Teoría y Métodos de Análisis de las Representaciones Sociales. En D. Paez (ed.) Pensamiento, Individuo y Sociedad. Cognición y Representación Social. Madrid. Fundamentos.

FI:Hr, B. (1988). Prototype Analysis of the concept of Love and Commitment. Journal of Personality and Social Psychology, 55, 557-579.

Ginsburg, G. (1988). Rules, Scripts and Prototypes in Personal Relationships. En S. Duck (Ed.) Handbook of Personal Relationships. Chichester. Wiley \& Sons.

Hallam, R. (1985). Anxiety. Londres. Academic Press.

Hendrick, C. (1988). Roles and Gender in relationships. En S. Duck (Ed.) Handbook of Personal Relationships. Chichester. Wiley \& Sons. 
Higgins, E. y Bargh, J. (1987). Social Cognition and Social Perception. Annual Review of Psychology. 38: 369-425.

HobFOL... S. y Stokes, J. (1988). The processes and mechanics of Social Support. En S. Duck (Ed.) Handbook of Personal Relationships. Chichester. Wiley \& Sons.

Odin. G. (1987). Concept, Knowledge, and Thought. Annual Review of Psychology. 38, 203227.

LAKOFF, G. y Kovecses. Z. (1987). The conceptualization of anger in American English. En N. Quinn y D. Holland (Eds.) Cultural Models in language and thought. Cambridge: Cambridge University Press.

LuTz, C. (1988). Ethnographics Perspectives on the Emotion Lexicon. En V. Hamilton, G. Bower \& N. Frijda. Cognitive Perspectives on Emotion and Motivation. Dordrecht. Kluwer Academic Press.

Nö̈L, M. P. y RIMÉ, B. (1988). Pensée operatoire, alexithymie et investigation psychosomatique: Revue Critique. European Bulletin of Cognitive Psychology, 8, 573-599.

Paez, D. et al. (1986). Salud Mental y Factores Psicosociales. Madrid. Fundamentos.

Paez, D., Echevarría, A. y Villareal, M. (1989). Teorías Sociopsicológicas de las Emociones. En A. Echevarría \& D. Paez (Eds.) Emociones: Perspectivas Psicosociales. Madrid. Fundamentos.

Paez, D. y Vergara, A. (1990). Procesos Psicosociales en la construcción del conocimiento social de las emociones. Sometido a publicación.

Pennebaker, J. (1988). Confiding Traumatic Experiences and Health. En S. Fisher y J. Reason (ed.) Handbook of Life Stress, Cognition and Health. Nueva York. Wiley \& Sons.

Pozo. J. (1989). Teorias Cognitivas del Aprendizaje. Madrid. Morata.

SAlMASO, P. y Pombeni, L. (1986). Le concept de travail. En W. Doise \& A. palmonari (Eds.) L'etude des Representations Sociales. Neuchatel. Delachaux-Niestlé.

SCHERER, K., RIMÉ, B. y CHIPP, P. (1989). L'expérience emotionnelle dans la culture européenne. En B. Rimé y K. Scherer (ed.) Les Emotions. Neuchatel. Delachaux-Niestlé.

Semin, G. (1989). Prototypes et Representations Sociales. En D. Jodelet (ed.) Representations Sociales. París. PUF.

ShAver, P. et al. (1987). Emotion knowledge: further exploration of a prototype approach. Journal of personality and Social Psychology. 52, 1061-1086.

THOITS, P. (1986). Multiples Identities: examining gender and marital status differences in distress. American Sociological Review. 51: 259-272.

Valencia, J., Paez, D. y Echevarria, A. (1989). Teorías Sociopsicológicas de las Emociones. En A. Echevarría y D. Paez (Eds.). Emociones: Perspectivas Psicosociales. Madrid. Fundamentos.

Vergara, A. y Paez, D. (1989). Diferencias Sexuales en Emocionalidad. En A. Echevarría y D. paez (Eds.). Emociones: Perspectivas Psicosociales. Madrid. Fundamentos.

Wood, W., Rhodes, N. y Whelan, M. (1989). Sex Differences in Positive Well-Being: A consideration of Emotional Style and Marital Status. Psychological Bulletin. 106. 